\title{
Safety and Efficacy of the Pipeline Embolization Device Use in the Outside Circle of Willis Located Intracranial Aneurysms: A Single-Center Experience
}

\author{
Sami Al Kasaba Waldo R. Guerrero a Daichi Nakagawab \\ Edgar A. Samaniego ${ }^{a-c}$ Santiago Ortega-Gutierrez ${ }^{a-c}$ David Hasan ${ }^{b}$ \\ aDepartment of Neurology, University of lowa Hospitals and Clinics, lowa City, IA, USA; \\ ${ }^{b}$ Department of Neurosurgery, University of lowa Hospitals and Clinics, lowa City, IA, USA; \\ 'Department of Radiology, University of lowa Hospitals and Clinics, lowa City, IA, USA
}

\section{Keywords}

Aneurysm · Circle of Willis · Off-label · Pipeline · Subarachnoid hemorrhage

\begin{abstract}
Background: The use of Pipeline Embolization Device (PED) is approved by the US Food and Drug Administration (FDA) to treat aneurysms located between the petrous and superior hypophyseal segments of the internal carotid artery. The purpose of this study is to evaluate the feasibility and efficacy of treating aneurysms outside the FDA approved anatomical locations. Furthermore, we analyze the safety of our antiplatelet protocol. Methods: Data on all patients treated with PED at our center from March 2015 to December 2017 were reviewed. Only patients with aneurysms treated with PED as off label use were included. Procedural complications and long-term functional outcome measured by modified Rankin Scale (mRS) were recorded. Tirofiban maintenance infusion was administered intravenously after PED deployment. None of the patients had platelet function testing. Results: A total of 36 patients harboring 36 aneurysms were included in the study. Mean age was 58.2 years \pm 14 .6. Nineteen were women (52.8\%) and most aneurysms were unruptured (80.6\%). There were no intraoperative complications. Five patients experienced postoperative complications -4 ischemic strokes and one groin hematoma. None led to any significant disability at follow-up. Thirty-one patients (86.1\%) achieved an mRS of 0-2 at follow-up. A Raymond-Roy classification of 1 was achieved in $78.9 \%$ of patients. Conclusion: The use of PED to treat aneurysms located outside the circle of Willis is feasible and effective. Our novel antiplatelet protocol did not require platelet function assay testing and did not lead to a higher rate of thrombo-embolic events compared to what has been previously reported.




\section{Introduction}

Flow diversion is a treatment option for anatomically challenging intracranial aneurysms and has been gaining increasing popularity among neuro-interventionists. The Pipeline Embolization Device (PED; ev3-Covidien, Irvine, CA, USA) is a self-expanding, braided cylindrical mesh consisting of 48 strands of cobalt-chromium and platinum-tungsten wires [1]. A unique feature that distinguishes PED from regular intracranial stents is the 30-35\% metal surface area coverage designed to assist endo saccular embolization [2]. Through this feature, the PED alters the hemodynamics within the aneurysm and its parent vessel and induces aneurysm thrombosis and eventually occlusion [3]. Following the publication of large multicenter trials [4, 5], PED was approved by the Food and Drug Administration (FDA) to treat large and giant unruptured aneurysms located between the petrous and superior hypophyseal segments of the internal carotid artery (ICA). While PED is FDA approved for use in that location, its use has expanded to include small aneurysms, ruptured aneurysms, and/or posterior circulation aneurysms. The new generation of PED, also called Pipeline Flex Embolization device, received FDA approval in 2015. In this study, we report the feasibility, and efficacy of off-label anatomical second-generation PED use for ruptured and unruptured intracranial aneurysms. Additionally, we report the safety profile and complication rates of PED with a novel periprocedural antiplatelet management protocol in the absence of preprocedural platelet activation testing.

\section{Materials and Methods}

The prospectively maintained data registry at the University of Iowa Hospitals and Clinics was analyzed. Patients with intracranial aneurysms in all arterial segments other than the segment between petrous, and superior hypophyseal segments were included in this study. Collected data included baseline characteristics, aneurysm characteristics, antiplatelet regimen, number of PEDs used, intraoperative and postoperative complications. Angiographic results post aneurysmal treatment with PED was assessed by DSA using the Raymond Roy occlusion Classification (RROC) where RROC class 1 indicates complete embolization, RROC class 2 indicates neck remanence, and RROC class 3 indicates the presence of residual aneurysm [6]. The study was approved by the Institutional Review Board.

\section{Statistical Analysis}

Normality was assessed for continuous variables using Shapiro-Wilk normality test. Student's $t$ test was used for normally distributed variables, whereas Mann-Whitney U test as a nonparametric alternative. In addition, Fisher exact or chi-square test was conducted to compare percentage of each of the binary groups. Postop complication was used a primary outcome. In all cases, a $p$ value of less than 0.05 was considered to indicate statistical significance. Statistical analysis was performed using GraphPad software version 7.03 (GraphPad Software).

\section{Results}

Patient Demographics and Aneurysm Characteristics

Between March 2015 and December 2017, 231 patients were treated with PED and of those 36 patients underwent off-label aneurysmal treatment with PED. Baseline characteristics are presented in Table 1. Mean age was $58.2 \pm 14.6,19$ patients were women $(52.8 \%)$, and 32 were Caucasian (88.9\%). Twenty-nine patients had modified Rankin Scale (mRS) of $0-2$ on admission, 3 had mRS of 4 , and 4 had mRS of 5. Seven aneurysms $(19.4 \%)$ were ruptured on presentation, and $29(80.6 \%)$ were unruptured. Thirty aneurysms were small $(<10 \mathrm{~mm}), 4$ were large $(10-25 \mathrm{~mm})$, and 2 were giant $(>25 \mathrm{~mm})$ aneurysms. The most 
Table 1. Patients' baseline characteristics

\begin{tabular}{lc}
\hline & Total $(n=36)$ \\
\hline Age, mean \pm SD & $58.2 \pm 14.6$ \\
Race, $n(\%)$ & $32(88.9)$ \\
$\quad$ White & $4(11.1)$ \\
$\quad$ Non-white & \\
Sex, $n$ (\%) & $19(52.8)$ \\
$\quad$ Female & $17(47.2)$ \\
$\quad$ Male & \\
Aneurysm location, $n(\%)$ & $6(16.7)$ \\
$\quad$ MCA & $7(19.4)$ \\
$\quad$ ACA & $2(5.6)$ \\
Acomm & $8(22.2)$ \\
Vertebral & $6(16.7)$ \\
Basilar & $7(19.4)$ \\
PCA & $7(19.4)$ \\
Ruptured, $n(\%)$ & $30(83.3)$ \\
Size & $4(11.1)$ \\
$\quad<10$ mm & $2(5.6)$ \\
10-25 mm &
\end{tabular}

MCA, middle cerebral artery; ACA, anterior cerebral artery; PCA, posterior cerebral artery; Acomm, anterior communicating artery.

common location was the vertebral artery (8/36), and most were intradural (7/8); this was followed by the anterior cerebral artery (ACA), and posterior cerebral artery $(7 / 36$ in each location), followed by middle cerebral artery (MCA; 6/36). Thirty-four aneurysms were treated for the first time and 2 were previously treated with primary coiling and pipeline stenting respectively. Table 2 demonstrates that pipeline complications were independent of age, sex, aneurysm size, mRS at admission, rupture status, and number of PEDs. Seven patients had 2 pipelines, one had 4 pipelines and the remainder patients had 1 pipeline placed. The number of devices was largely dependent on the size of the aneurysm.

\section{Endovascular Procedure}

All elective procedures were performed under minimal conscious sedation. An access guide catheter was used to selectively catheterize the major parent vessel (ICA, vertebral) under fluoroscopic guidance. A Marksman microcatheter (Medtronic) was then advanced distal to the aneurysm using a Synchro-2 microwire (Stryker) under roadmap. Depending on the aneurysm size, 1, 2, or 4 PEDs (Medtronic) were deployed through the Marksman microcatheter using a coaxial guide-catheter system.

\section{Antiplatelet Regimen}

For unruptured aneurysms, patients were bolused with 3,000 units of heparin at procedure onset. Patients with ruptured aneurysms did not receive a heparin bolus. Tirofiban (Medicure) was administered immediately following the deployment of the PED: no bolus was given and an infusion of $0.1 \mu \mathrm{g} / \mathrm{kg} / \mathrm{min}$ was maintained for 2-6 h after the procedure as described previously $[7,8]$. All patients were also loaded with dual antiplatelet (aspirin 325 $\mathrm{mg}$ and clopidogrel $600 \mathrm{mg}$ ) immediately pre or post procedure. Aspirin and clopidogrel response testing was not performed pre or post procedure. Most aneurysms (28, [78\%]) were treated with 1 pipeline, 7 aneurysms required 2 pipeline devices, and 1 required 4 pipeline devices. 
Table 2. Relationship between patients and aneurysm features and complication rates

\begin{tabular}{|c|c|c|c|}
\hline & \multicolumn{2}{|c|}{ Post-complication } & \multirow[t]{2}{*}{$p$ value } \\
\hline & positive $(n=5)$ & negative $(n=31)$ & \\
\hline Age, mean \pm SD & $60.0 \pm 12.1$ & $57.9 \pm 15.4$ & 0.77 \\
\hline Female, $n(\%)$ & $2(40.0)$ & $16(51.6)$ & $>0.99$ \\
\hline Aneurysm size, mm, median (IQR) & 3.0 & 5.0 & 0.31 \\
\hline Aneurysm location $n(\%)$ & & & 0.66 \\
\hline MCA & $1(20.0)$ & $5(16.1)$ & \\
\hline ACA & $2(40.0)^{*}$ & $5(16.1)$ & \\
\hline Acomm & $0(0)$ & $2(6.5)$ & \\
\hline Vertebral & $0(0)$ & $9(29.0)$ & \\
\hline Basilar & $1(20.0)$ & $4(12.9)$ & \\
\hline PCA & $1(20.0)$ & $6(19.4)$ & \\
\hline mRS at admission, median (IQR) & 0 & 0 & 0.33 \\
\hline Ruptured, $n(\%)$ & $0(0)$ & $7(22.6)$ & 0.56 \\
\hline Number of pipeline devices, median (IQR) & 1 & 1 & $>0.99$ \\
\hline
\end{tabular}

* One of 2 complications in ACA was groin hematoma.

MCA, middle cerebral artery; ACA, anterior cerebral artery; PCA, posterior cerebral artery; Acomm, anterior communicating artery; IQR, interquartile range.

\section{Complications and Outcomes}

No intraoperative complications were reported. Five patients (13.9\%) experienced postoperative complications: Four ischemic strokes and 1 groin hematoma (Fig. 1). One patient with an anterior communicating artery (Acomm) aneurysm experienced mild left-sided weakness following PED placement, MRI brain showed right MCA territory infarct. The patient's left-sided weakness improved upon discharge and was at baseline at 3 months' follow-up. Another patient developed right-sided weakness following the procedure, and at follow-up, the patient had very mild right leg weakness. The third patient presented with multiple brainstem infarcts and found to have a giant thrombosed basilar artery aneurysm. This patient expired during his hospital stay. He initially presented with left-sided weakness, and shortly after that he started having decreased level of consciousness and was intubated for airway protection. He underwent PED deployment in his basilar artery; however, given the lack of exam improvement, the family elected to pursue comfort care. The fourth patient had sudden onset right face and arm numbness 1 day following PED placement. She was found to have left P1 occlusion and underwent mechanical thrombectomy. The patient was discharged home with no focal neurologic deficit. Three of the 9 (33.3\%) patients with PED deployed in the ACA territory (A1 segment) had ischemic stroke and 2/21 (9.5\%) in the posterior circulation. Nineteen patients had follow-up angiograms, 15 (78.9\%) of whom had Raymond-Roy scale of 1, $3(15.8 \%)$ achieved a scale of 2 , and $1(5.3 \%)$ had a score of 3 (Fig. 2-4). Time of the follow-up angiogram was between 3 and 8 months with an average of 5.9 months following PED placement. For the remaining 17 patients, 9 patients had a followup arterial imaging with MRA or CTA of the head. Of those, 6 showed no residual aneurysm, 1 showed tiny residual aneurysm, and 2 had residual aneurysm. All patients were started on aspirin $325 \mathrm{mg}$ and clopidogrel $75 \mathrm{mg}$ daily at the beginning of the procedure or immediately post procedure. Two patients were eventually switched from clopidogrel to ticagrelor after experiencing ischemic stroke complication. We empirically switched these 2 patients and did not perform platelet function before changing their antiplatelet regimen. At follow-up, 31 patients $(86.1 \%)$ achieved an mRS of $0-2$. 


\section{Interventional \\ Neurology}

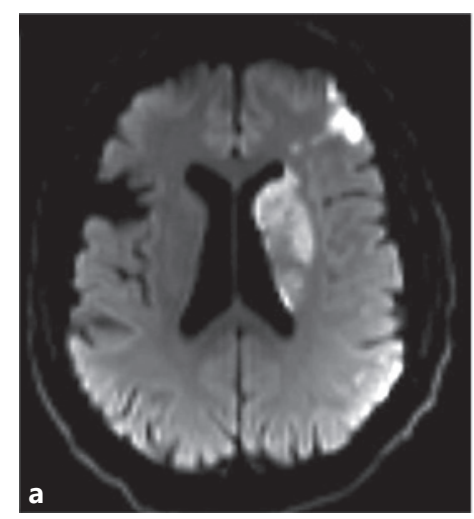

\begin{tabular}{l|l}
\hline Intervent Neurol 2019;8:83-91 \\
\hline DOI: 10.1159/000495074 & $\begin{array}{l}\text { @ 2 2019 S. Karger AG, Basel } \\
\text { www.karger.com/ine }\end{array}$ \\
\hline
\end{tabular}

Al Kasab et al.: Safety and Efficacy of the PED Use in Outside Circle of Willis Located Intracranial Aneurysms: A Single-Center Experience
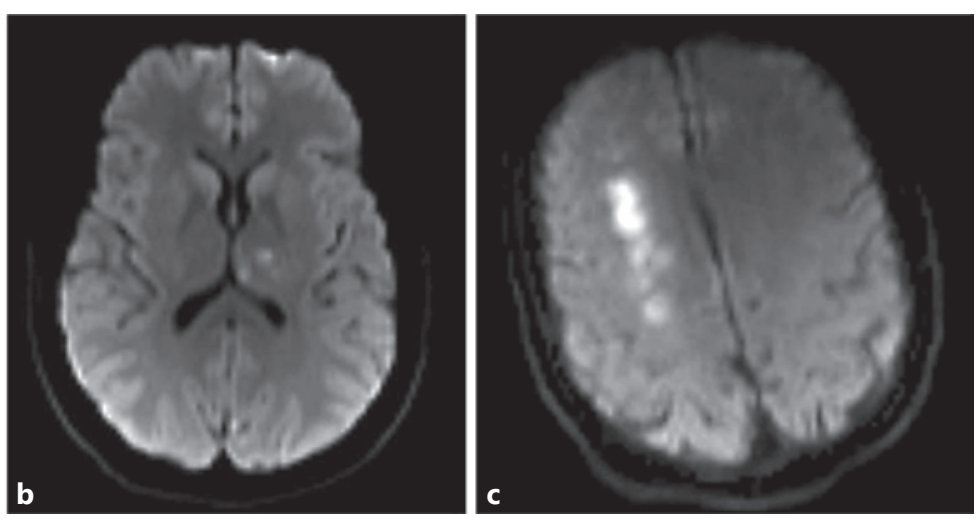

Fig. 1. MRI diffusion-weighted imaging of 3 out of the 4 patients who experienced ischemic strokes in the left basal ganglia and frontal lobe (a), left thalamus (b), right MCA-ACA watershed zone (c) following PED placement.
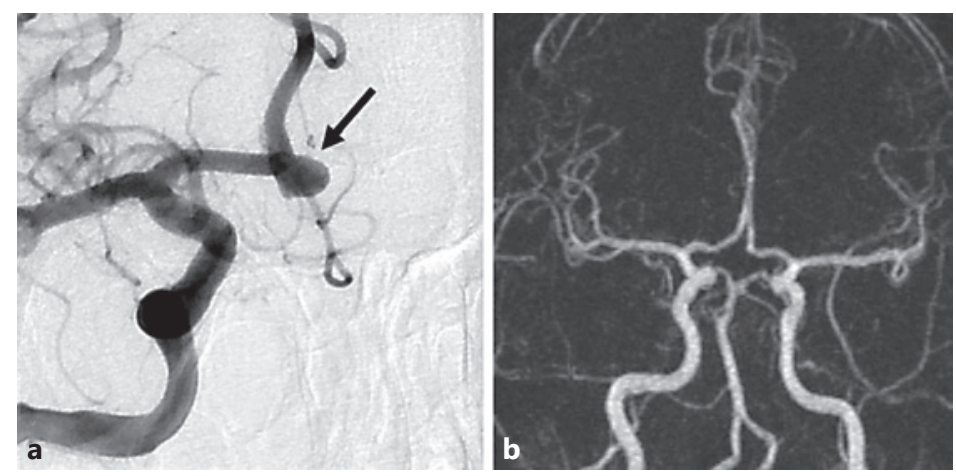

Fig. 2. The illustrative case of a middle aged woman with no past medical history who was found to have a 5 $\times 4.4 \mathrm{~mm}$ ACoA aneurysm on an MRA done for workup of headache. The patient underwent elective coil and pipeline embolization of his aneurysm (a; black arrow). The procedure was uncomplicated and patient was discharged the next day. MRA done 6 months later showed no recurrence of the aneurysm (b).
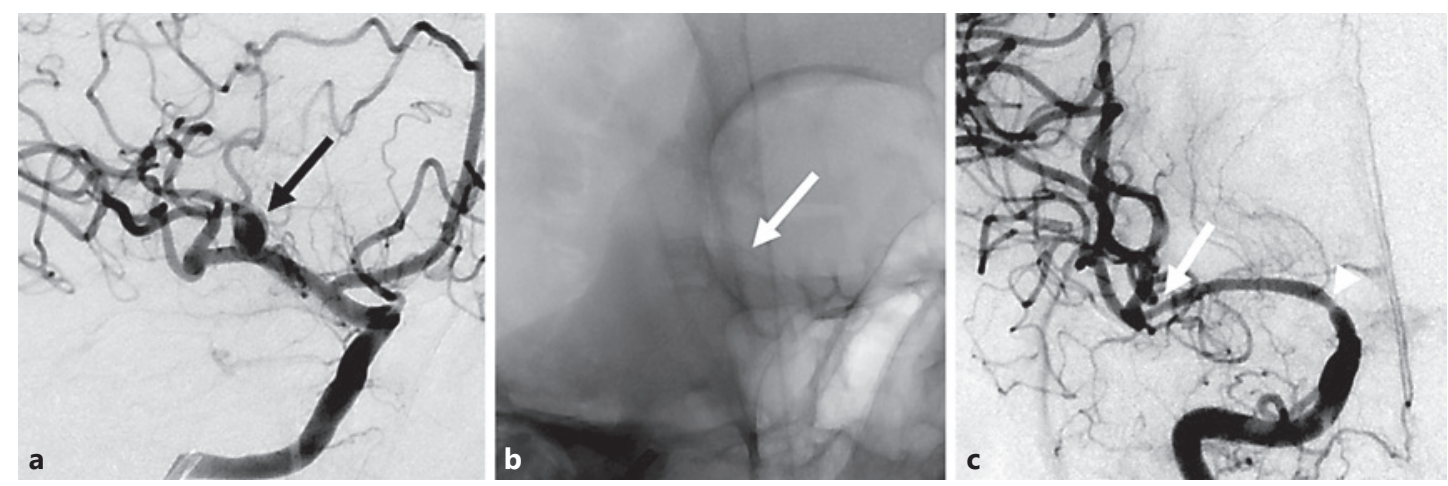

Fig. 3. A man in his middle age with a history of COPD was found to have a right MCA aneurysm on an MRA done for workup of syncope. Patient underwent pipeline embolization of the right MCA aneurysm (black arrow) without complications (a). He was discharged home the next day. b Native imaging showing pipeline placement (white arrow). Follow-up DSA 6 months later showed no recurrence of the aneurysm with mild intimal hyperplasia at the proximal (white arrowhead) and distal end (white arrow) of the stent and minimal filling of the right ACA (c). 
Al Kasab et al.: Safety and Efficacy of the PED Use in Outside Circle of Willis Located Intracranial Aneurysms: A Single-Center Experience

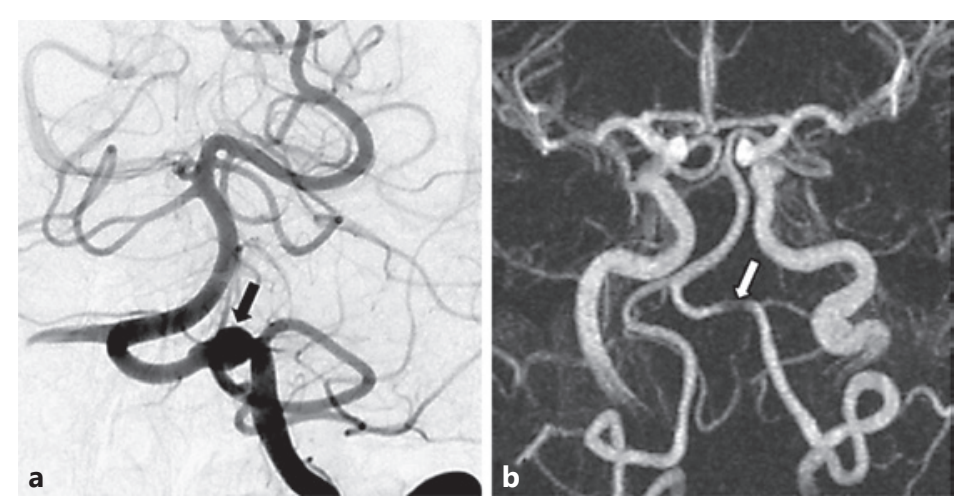

Fig. 4. A middle-aged man who was found to have a left VA aneurysm while being worked up for headache. He underwent DSA that showed a fusiform aneurysm (black arrow) just distal the PICA (a). Patient underwent pipeline embolization of the aneurysm. Follow-up MRA 6 months later demonstrated that no residual aneurysm is seen. There is flow through the stented segment with a small amount of in-stent signal loss (white arrow), which was felt to be either artifactual or related to in-stent stenosis (b).

\section{Discussion}

Our case series suggests that using pipeline flow diverting devices to treat intracranial aneurysms outside the circle of Willis may be feasible and effective. Importantly, our study demonstrates that our antiplatelet regimen, which does not require platelet activation testing prior to or after PED use was not associated with higher complication rates when compared to previous studies that used platelet function testing. The complication rate observed in our case series falls well with the previously reported complication rate with use of PED in the FDA approved anatomical location [9].

The safety and efficacy of the first-generation PED have been shown in multiple previous clinical trials leading to its approval by the FDA in 2014 [5, 9, 10]. The IntrePED (International Retrospective Study of the PED) study was a multicenter, retrospective study that assessed the complication rates following PED placement for intracranial aneurysm treatment in a real-world setting. In this study, most aneurysms were in the ICA and only $6.5 \%$ were in the posterior circulation and $1.7 \%$ in the MCA. The complication rate was $8.4 \%$. Most of complications were related to ischemic stroke. Our study shows similar rate of complications; however, our study is unique in that we only included patients with aneurysms located outside the FDA-approved locations.

Aneurysms of the MCA, ACA, and ACoA account for 5-10\% of aneurysms [11]. The current gold standard treatment for such aneurysms is microsurgical clipping [11,12]. While microsurgical clipping is associated with high occlusion rates, it has increased morbidity and mortality $[11,12]$. Given the small size of the parent artery of such aneurysms are small $(<2.5$ $\mathrm{mm}$ in caliber), endovascular treatment can be challenging [13]. Stenosis, parent vessel occlusion secondary to neointimal hyperplasia/inflammatory response, and branch vessel occlusion are some of the complications related to PED placement [14]. Few previous studies evaluated the use of PED for aneurysms in the distal anterior circulation location. A study by Lin et al. [15] evaluated 28 patients with distal anterior circulation aneurysms treated with PED. The complication rate during the periprocedural period was $10.7 \%$, and no deaths were reported in this study. Most patients (96.4\%) had a good outcome, with mRS scores between 0 and 2, and 1 patient had a fair outcome, with an mRS score of 3. Martínez-Galdámez et al. [16] also looked at the use of PED for treatment of 25 aneurysms outside the circle of Willis. 
In this series, there were 2 minor postoperative events, both resolved spontaneously within $24 \mathrm{~h}, 1$ major postoperative event, as well as 3 procedural complications that resolved without major deficits. No deaths were reported in this study either. Our study findings are in concurrence with those of other studies and demonstrate promising results for the use of the PED for the treatment of aneurysms in the distal cerebrovascular circulation, and more importantly in cases that are considered too difficult to treat using conventional methods.

The use of the PED in the posterior circulation has not been widely accepted due to the unique characteristics of the cerebral vasculature and aneurysms arising in this location. Specifically, numerous unforgiving perforator vessels arise in this area and supply brainstem structures that could become occluded and could lead to significant disability $[17,18]$. Previous studies evaluating the use of the PED in the posterior circulation have demonstrated higher mortality and morbidity rates [18, 19]. Phillips et al. [18] assessed the safety of PED placement in 32 patients with posterior circulation aneurysms. Of the 21 patients who had basilar artery aneurysms, perforator infarctions were seen in 3 patients (14\%), although just a single PED was used in each case. The authors concluded that clinical perforator infarction rates may be higher when the PED is placed within the basilar artery compared with the ICA. More recent studies have demonstrated better outcomes. Munich et al. [17] assessed the outcomes in 12 patients with vertebrobasilar fusiform aneurysms treated with the PED. Complications related to the procedure occurred in 3 patients, resulting in death in 1 of the cases; the other 2 cases had significant improvement of symptoms. There were no thromboembolic complications seen in this series. More recently, our group published a series of 7 patients treated for ruptured vertebrobasilar dissecting aneurysms [20]. In our series, there were no intraoperative complications directly related to the PED placement and no patient re-bled. In this study, 21 patients had posterior circulation aneurysms. Only 2 patients experienced a complication. These patients had an ischemic stroke that did not lead to long-term disability upon follow-up.

Thromboembolic events after PED placement remains a feared complication. Previous studies reported the presence of diffusion-weighted imaging changes on MRI in up to $52 \%$ of patients treated with PED [21]. To reduce the risk thromboembolism associated with PED, antiplatelet agents are routinely used following PED placement [22]. The most widely utilized regiment is a combination of aspirin and clopidogrel. Evidence from cardiology literature suggests that antiplatelet regimen modification based on platelet function tests might be associated with better clinical outcome [23-25]. Many neuro-interventionalists use platelet function assays to guide their antiplatelet regimen; however, there is paucity of evidence in the neuro-endovascular literature. Controversy exists as to whether platelet testing is necessary in patients who undergo PED placement because the benefits are yet to be proven. Brinjikji et al. [26] used the International Retrospective Study of PED registry and studied the complication rates in groups of patients who underwent platelet testing and those who did not undergo platelet testing. After evaluating 800 patients they found that platelet function testing was associated with significantly higher odds of morbidity in patients who underwent PED placement. The authors speculated that aggressive periprocedural manipulation of loading doses of antiplatelet or other adjunctive drugs may be causal. In 2016, Skukalek et al. [27] published an extensive review and meta-analysis examining the impact of platelet testing in patients treated with PED on the rates of thrombotic and hemorrhagic complications. Their analysis of 19 single-center and multicenter studies found no significant association between the use of platelet function test and the rates of symptomatic thrombotic or hemorrhagic complications with PED placement. In our study, our patients did not have a platelet function test prior to the procedure as the decision to use PED was made intraoperatively after carefully considering alternative options. In those cases, the interventionist determined that alternatives were not viable and the decision was then made to proceed with PED. We utilized 
our recently published protocol for antiplatelet where we used tirofiban during flow diversion [8]. Patients were loaded pre-procedure with aspirin $325 \mathrm{mg}$ and clopidogrel $600 \mathrm{mg}$ immediately before PED placement if unruptured aneurysm and post procedure if ruptured aneurysm. In addition to using this novel regimen, we did not perform platelet function testing to guide long-term antiplatelet regimen. The rate of thrombo-embolic events in our series is similar to what has been reported in previous studies with PED despite the use of platelet function assay. These findings are important because they suggest that preoperative platelet testing does not result in improved outcomes of patients who undergo PED placement.

\section{Limitations}

This study includes a very small series and extreme caution must be employed and strong recommendation not to abandon the use of platelet function assay testing.

\section{Conclusion}

The use of PED to treat aneurysms outside the circle of Willis is safe and effective. Further prospective studies are warranted to confirm our findings.

\section{Acknowledgment}

None.

\section{Funding Source}

This work was partially supported by funding from Nakatani Foundation.

\section{Disclosure Statement}

The authors have no personal financial or institutional interest in any of the drugs, materials, or devices described in this article.

\section{References}

1 Fischer S, Vajda Z, Aguilar Perez M, Schmid E, Hopf N, Bäzner H, et al. Pipeline embolization device (PED) for neurovascular reconstruction: initial experience in the treatment of 101 intracranial aneurysms and dissections. Neuroradiology. 2012 Apr;54(4):369-82.

2 Alderazi YJ, Shastri D, Kass-Hout T, Prestigiacomo CJ, Gandhi CD. Flow diverters for intracranial aneurysms. Stroke Res Treat. 2014;2014:415653.

3 Fiorella D, Lylyk P, Szikora I, Kelly ME, Albuquerque FC, McDougall CG, et al. Curative cerebrovascular reconstruction with the Pipeline embolization device: the emergence of definitive endovascular therapy for intracranial aneurysms. J Neurointerv Surg. 2009 Jul;1(1):56-65.

4 Nelson PK, Lylyk P, Szikora I, Wetzel SG, Wanke I, Fiorella D. The pipeline embolization device for the intracranial treatment of aneurysms trial. AJNR Am J Neuroradiol. 2011 Jan;32(1):34-40.

5 Becske T, Kallmes DF, Saatci I, McDougall CG, Szikora I, Lanzino G, et al. Pipeline for uncoilable or failed aneurysms: results from a multicenter clinical trial. Radiology. 2013 Jun;267(3):858-68.

6 Roy D, Milot G, Raymond J. Endovascular treatment of unruptured aneurysms. Stroke. 2001 Sep;32(9):19982004.

7 Chalouhi N, Jabbour P, Kung D, Hasan D. Safety and efficacy of tirofiban in stent-assisted coil embolization of intracranial aneurysms. Neurosurgery. 2012 Sep;71(3):710-4.

8 Chalouhi N, Jabbour P, Daou B, Starke RM, Shields B, Hasan DM. A New Protocol for Anticoagulation With Tirofiban During Flow Diversion. Neurosurgery. 2016 May;78(5):670-4. 
9 Kallmes DF, Brinjikji W, Boccardi E, Ciceri E, Diaz O, Tawk R, et al.; Aneurysm Study of Pipeline in an Observational Registry. ASPIRe. Interv Neurol. 2016 Jun; 5(1-2): 89-99.

10 Kallmes DF, Hanel R, Lopes D, Boccardi E, Bonafé A, Cekirge S, et al. International retrospective study of the pipeline embolization device: a multicenter aneurysm treatment study. AJNR Am J Neuroradiol. 2015 Jan; 36(1):108-15.

11 Nossek E, Zumofen DW, Setton A, Potts MB, Raz E, Shapiro M, et al. Treatment of distal anterior cerebral artery aneurysms with the Pipeline Embolization Device. J Clin Neurosci. 2017 Jan;35:133-8.

12 Vora N, Thomas AJ, Gupta R, Gologorsky Y, Panapitiya N, Jovin T, et al. Endovascular treatment of distal anterior cerebral artery aneurysms: technical results and review of the literature. J Neuroimaging. 2010 Jan; 20(1):70-3.

13 Murthy SB, Shah J, Mangat HS, Stieg P. Treatment of Intracranial Aneurysms With Pipeline Embolization Device: Newer Applications and Technical Advances. Curr Treat Options Neurol. 2016 Apr;18(4):16.

14 Durst CR, Hixson HR, Schmitt P, Gingras JM, Crowley RW. Endovascular Treatment of a Fusiform Aneurysm at the M3-M4 Junction of the Middle Cerebral Artery Using the Pipeline Embolization Device. World Neurosurg. 2016 Feb;86:511.e1-4.

15 Lin N, Lanzino G, Lopes DK, Arthur AS, Ogilvy CS, Ecker RD, et al. Treatment of Distal Anterior Circulation Aneurysms With the Pipeline Embolization Device: A US Multicenter Experience. Neurosurgery. 2016 Jul; 79(1):14-22.

16 Martínez-Galdámez M, Romance A, Vega P, Vega A, Caniego JL, Paul L, et al. Pipeline endovascular device for the treatment of intracranial aneurysms at the level of the circle of Willis and beyond: multicenter experience. J Neurointerv Surg. 2015 Nov;7(11):816-23.

17 Munich SA, Tan LA, Keigher KM, Chen M, Moftakhar R, Lopes DK. The Pipeline Embolization Device for the treatment of posterior circulation fusiform aneurysms: lessons learned at a single institution. J Neurosurg. 2014 Nov;121(5):1077-84.

18 Phillips TJ, Wenderoth JD, Phatouros CC, Rice H, Singh TP, Devilliers L, et al. Safety of the pipeline embolization device in treatment of posterior circulation aneurysms. AJNR Am J Neuroradiol. 2012 Aug;33(7):1225-31.

19 Siddiqui AH, Abla AA, Kan P, Dumont TM, Jahshan S, Britz GW, et al. Panacea or problem: flow diverters in the treatment of symptomatic large or giant fusiform vertebrobasilar aneurysms. J Neurosurg. 2012 Jun;116(6): 1258-66.

20 Guerrero WR, Ortega-Gutierrez S, Hayakawa M, Derdeyn CP, Rossen JD, Hasan D, et al. Endovascular Treatment of Ruptured Vertebrobasilar Dissecting Aneurysms Using Flow Diversion Embolization Devices: Single-Institution Experience. World Neurosurg. 2018 Jan;109:e164-9.

21 Heller RS, Dandamudi V, Lanfranchi M, Malek AM. Effect of antiplatelet therapy on thromboembolism after flow diversion with the pipeline embolization device. J Neurosurg. 2013 Dec;119(6):1603-10.

22 Adeeb N, Griessenauer CJ, Foreman PM, Moore JM, Shallwani H, Motiei-Langroudi R, et al. Use of Platelet Function Testing Before Pipeline Embolization Device Placement: A Multicenter Cohort Study. Stroke. 2017 May;48(5):1322-30.

23 Price MJ, Angiolillo DJ, Teirstein PS, Lillie E, Manoukian SV, Berger PB, et al. Platelet reactivity and cardiovascular outcomes after percutaneous coronary intervention: a time-dependent analysis of the Gauging Responsiveness with a VerifyNow P2Y12 assay: Impact on Thrombosis and Safety (GRAVITAS) trial. Circulation. 2011 Sep;124(10):1132-7.

24 Price MJ, Berger PB, Angiolillo DJ, Teirstein PS, Tanguay JF, Kandzari DE, et al. Evaluation of individualized clopidogrel therapy after drug-eluting stent implantation in patients with high residual platelet reactivity: design and rationale of the GRAVITAS trial. Am Heart J. 2009 May;157(5):818-24.

25 Bonello L, Camoin-Jau L, Armero S, Com O, Arques S, Burignat-Bonello C, et al. Tailored clopidogrel loading dose according to platelet reactivity monitoring to prevent acute and subacute stent thrombosis. Am J Cardiol. 2009 Jan;103(1):5-10.

26 Brinjikji W, Lanzino G, Cloft HJ, Siddiqui AH, Hanel RA, Kallmes DF. Platelet Testing is Associated with Worse Clinical Outcomes for Patients Treated with the Pipeline Embolization Device. AJNR Am J Neuroradiol. 2015 Nov;36(11):2090-5.

27 Skukalek SL, Winkler AM, Kang J, Dion JE, Cawley CM, Webb A, et al. Effect of antiplatelet therapy and platelet function testing on hemorrhagic and thrombotic complications in patients with cerebral aneurysms treated with the pipeline embolization device: a review and meta-analysis. J Neurointerv Surg. 2016 Jan;8(1):58-65. 